\title{
Beryllium abundances along the evolutionary sequence of the open cluster IC 4651 - A new test for hydrodynamical stellar models ${ }^{\star}$
}

\author{
R. Smiljanic ${ }^{1, \star \star}$, L. Pasquini ${ }^{2}$, C. Charbonnel ${ }^{3,4}$, and N. Lagarde ${ }^{3}$ \\ 1 Universidade de São Paulo, IAG, Dep. de Astronomia, Rua do Matão 1226, São Paulo-SP 05508-090, Brazil \\ e-mail: rsmiljan@eso.org \\ 2 European Southern Observatory, Karl-Schwarzschild-Str. 2, 85748 Garching bei München, Germany \\ e-mail: lpasquin@eso.org \\ 3 Geneva Observatory, University of Geneva, chemin des Maillettes 51, 1290 Versoix, Switzerland \\ e-mail: [Corinne.Charbonnel; Nadege.Lagarde]@unige.ch \\ ${ }^{4}$ LATT, CNRS UMR 5572, Université de Toulouse, 14 avenue Edouard Belin, 31400 Toulouse Cedex 04, France
}

Received 22 July 2009 / Accepted 5 November 2009

\begin{abstract}
Context. Previous analyses of lithium abundances in main sequence and red giant stars have revealed the action of mixing mechanisms other than convection in stellar interiors. Beryllium abundances in stars with Li abundance determinations can offer valuable complementary information on the nature of these mechanisms.

Aims. Our aim is to derive Be abundances along the whole evolutionary sequence of an open cluster. We focus on the well-studied open cluster IC 4651 . These Be abundances are used with previously determined Li abundances, in the same sample stars, to investigate the mixing mechanisms in a range of stellar masses and evolutionary stages.

Methods. Atmospheric parameters were adopted from a previous abundance analysis by the same authors. New Be abundances have been determined from high-resolution, high signal-to-noise UVES spectra using spectrum synthesis and model atmospheres. The careful synthetic modeling of the Be lines region is used to calculate reliable abundances in rapidly rotating stars. The observed behavior of $\mathrm{Be}$ and $\mathrm{Li}$ is compared to theoretical predictions from stellar models including rotation-induced mixing, internal gravity waves, atomic diffusion, and thermohaline mixing.

Results. Beryllium is detected in all the main sequence and turn-off sample stars, both slow- and fast-rotating stars, including the Li-dip stars, but is not detected in the red giants. Confirming previous results, we find that the Li dip is also a Be dip, although the depletion of $\mathrm{Be}$ is more modest than for $\mathrm{Li}$ in the corresponding effective temperature range. For post-main-sequence stars, the $\mathrm{Be}$ dilution starts earlier within the Hertzsprung gap than expected from classical predictions, as does the Li dilution. A clear dispersion in the Be abundances is also observed. Theoretical stellar models including the hydrodynamical transport processes mentioned above are able to reproduce all the observed features well. These results show a good theoretical understanding of the $\mathrm{Li}$ and Be behavior along the color-magnitude diagram of this intermediate-age cluster for stars more massive than $1.2 M_{\odot}$.
\end{abstract}

Key words. stars: abundances - stars: evolution - rotation - open clusters and associations: individual: IC 4651

\section{Introduction}

The classical theory of stellar evolution, which only allows for mixing in stellar convective layers, fails to explain nearly all $\mathrm{Li}$ abundance patterns observed so far at the surface of low-mass stars. Indeed, in contradiction to the classical predictions, the vast majority of F- and early G-type stars (including the Sun) deplete, often quite severely, their surface $\mathrm{Li}$ abundance during their main-sequence lifetime, revealing transport processes beyond convection. A-type stars are also concerned, although in these objects the signatures of non-standard Li depletion, occurring in their interior during the main sequence, appear at their

* Based on observations made with the ESO VLT, at Paranal Observatory, under programs 065.L-0427 and 067.D-0126.

$\star \star$ Current address: European Southern Observatory, KarlSchwarzschild-Str. 2, 85748 Garching bei München, Germany. surface only when they cross the Hertzsprung gap and become subgiants.

Lithium depletion is seen both in field (e.g., Herbig 1965; Duncan 1981; Pasquini et al. 1994; Lèbre et al. 1999; Chen et al. 2001; Lambert \& Reddy 2004, and references therein) and in open cluster stars (e.g. Wallerstein et al. 1965; Zappala 1972; Cayrel et al. 1984; Soderblom et al. 1990; Balachandran 1995; Jones et al. 1997; Pasquini et al. 2001; Sestito \& Randich 2005, and references therein). As cluster stars have well-defined masses and, most important, share the same age and initial chemical composition, they are ideal targets for investigating mixing processes as a function of stellar mass and evolutionary status. They have therefore received considerable attention in the literature. Observations of the Li evolution along the color magnitude diagram (CMD) of open clusters have revealed a number of interesting features, such as the Li-dip (Wallerstein et al. 1965; Boesgaard \& Tripicco 1986), the lack of abundance trends with 
age among A-type stars (Burkhart \& Coupry 1998, 2000), the presence of a spread of $\mathrm{Li}$ among solar-type stars in relatively old clusters like M67 (Pasquini et al. 1997; Jones et al. 1999), and the Li variations with mass and evolutionary status as in the case of IC 4651 (Pasquini et al. 2004).

Many different physical mechanisms have been proposed to explain these observations: atomic diffusion (Michaud 1986; Michaud et al. 2004), mass loss (Hobbs et al. 1989; Swenson \& Faulkner 1992), rotation-induced mixing (Charbonnel et al. 1992; Deliyannis \& Pinsonneault 1997; Talon \& Charbonnel 1998; Palacios et al. 2003; Théado \& Vauclair 2003), mixing by internal gravity waves (Garcia Lopez \& Spruit 1991; Montalbán \& Schatzman 2000; Young et al. 2003), or a combination of some of these processes. So far, only the hydrodynamic stellar models that combine the effects of meridional circulation, shear turbulence, internal gravity waves, and atomic diffusion have been able to account for $\mathrm{Li}$ observations over a broad range of stellar masses, ages, and evolutionary status (Talon \& Charbonnel 2003, 2005; Charbonnel \& Talon 2005, 2008). These models, which use a prescription for the wave excitation that reproduces the solar-p modes (Goldreich et al. 1994), explain other observational constraints concomitantly, like the behavior of $\mathrm{C}, \mathrm{N}, \mathrm{O}$, and $\mathrm{Be}$ across the $\mathrm{Li}$ dip in young clusters, such as the Hyades, or the internal rotation profile of the Sun (Charbonnel \& Talon 2005).

Additional clues can help ascertain the nature and description of transport mechanisms of chemicals and angular momentum inside low-mass stars. In particular the simultaneous determination of $\mathrm{Li}$ and $\mathrm{Be}$ abundances in the same stars strengthens the constraints by performing a stellar tomography. Such a powerful diagnosis is possible because $\mathrm{Li}$ and $\mathrm{Be}$ burn at different temperatures $\left(\sim 2.5 \times 10^{6} \mathrm{~K}\right.$ for $\mathrm{Li}$ and $\sim 3.5 \times 10^{6} \mathrm{~K}$ for $\left.\mathrm{Be}\right)$, which correspond to different depths in the stellar interior (see e.g. Deliyannis et al. 2000).

Although the simultaneous study of $\mathrm{Li}$ and $\mathrm{Be}$ in the same stars is a powerful tool, Be has been determined in far fewer stars than Li has. In particular, despite the advantages of cluster stars mentioned above, Be has been investigated in only a few open clusters (e.g. Boesgaard et al. 1977, 2004a; Garcia Lopez et al. 1995; Randich et al. 2002, 2007, and references therein), while field stars have been more extensively studied (e.g. Boesgaard 1976; Stephens et al. 1997; Santos et al. 2004; Boesgaard \& Krugler Hollek 2009, and references therein).

Boesgaard and collaborators discovered a Be dip and a correlation between $\mathrm{Li}$ and $\mathrm{Be}$ depletion in mid F-stars in the field (Deliyannis et al. 1998) and also in the Hyades, Coma, and Praesepe clusters (Boesgaard \& King 2002; Boesgaard et al. 2004a). They also show that for cooler $G$ stars there is little or no Be depletion, even in Li-depleted objects up to $\sim 5500 \mathrm{~K}$ (Boesgaard et al. 2004b, and references therein). For even cooler stars, below $~ 5500 \mathrm{~K}$, Santos et al. (2004) show a Be decline correlated with decreasing temperature. These findings were confirmed by Randich et al. (2002, 2007), who investigated Li and $\mathrm{Be}$ in late F- and early G-type stars in some open clusters over a relatively broad age range (IC 2391 - 50 Myr, NGC 2516 150 Myr, Hyades - 600 Myr, IC 4651 - 1.7 Gyr, and M 67 4.5 Gyr).

To improve our understanding of the mixing processes, adding new Be data covering a large fraction of the color magnitude diagram of well-studied open clusters is important. The ambitious aim is to compare the observations with models for stars over as wide as possible a range of mass and evolutionary status. In this context, the open cluster IC 4651 is a very good test case. It has well-determined membership

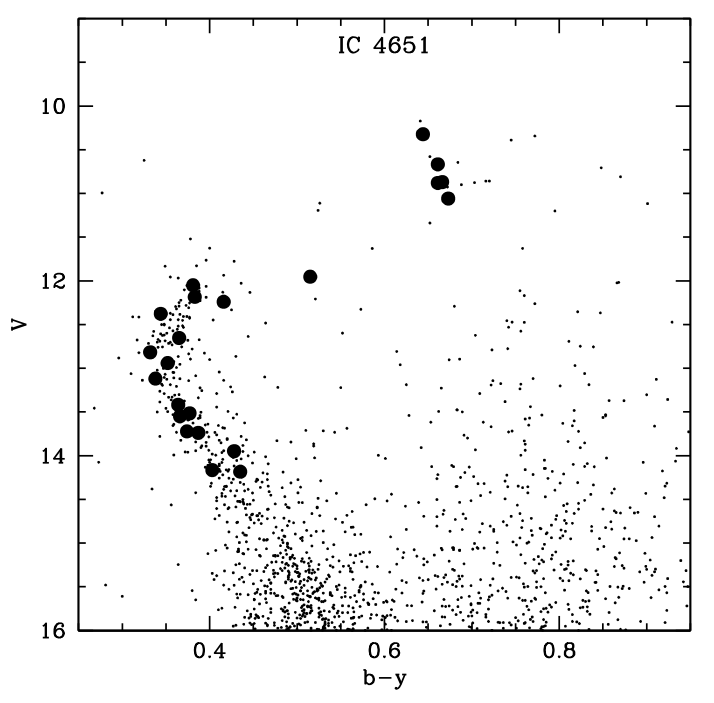

Fig. 1. Color magnitude diagram of IC 4651. Sample stars are shown as filled circles. The $u b v y$ photometry is from Meibom (2000) and obtained by means of the WEBDA database.

(Meibom et al. 2002), and precisely known metallicity and chemical composition $([\mathrm{Fe} / \mathrm{H}]=+0.11$; Pasquini et al. 2004; Carretta et al. 2004; Pace et al. 2008). Age determinations for this cluster vary between $1.2 \pm 0.2$ Gyr (Biazzo et al. 2007) and $1.7 \pm 0.15$ Gyr (Meibom et al. 2002). A color magnitude diagram of IC 4651 is shown in Fig. 1.

Important is that the $\mathrm{Li}$ evolution along the $\mathrm{CMD}$ of this cluster has already been investigated in detail by Pasquini et al. (2004). Well-determined patterns have been found along its evolutionary sequence (see Fig. 3 by Pasquini et al. 2004). In a sequence of increasing stellar mass, the cluster indeed has an $\mathrm{Li}$ "plateau" around solar mass stars, followed by a welldetermined Li dip, after which the maximum Li content is observed, at meteoritic value. A sudden $\mathrm{Li}$ drop follows along the Hertzsprung gap, possibly indicating the onset of dilution (the so-called first dredge-up). Some of the giants show noticeable differences in $\mathrm{Li}$ abundance while sharing other stellar characteristics. It has been shown that models of rotating stars by Charbonnel \& Talon (1999) and Palacios et al. (2003) were able to reproduce the $\mathrm{Li}$ behavior both of main sequence stars lying on the blue side of the dip and of evolved stars.

In this work we present Be abundances for the same stars analyzed by Pasquini et al. (2004). This is the first time Be abundances have been determined for stars along the whole evolutionary sequence of an open cluster, including solar-type, Li-dip, turn-off, subgiant, and red giant stars. With these new results, the mixing processes in different stellar masses can be investigated in greater detail.

\section{Observations and stellar parameters}

Our sample is composed of 22 stars. Twenty one of them have been analyzed by Pasquini et al. (2004). To these we add one star of this same cluster analyzed by Randich et al. (2002), star T2105. The sample was selected to cover a large interval of the CMD from the Strömgren photometry obtained by Meibom (2000). The interested reader is referred to the work of Pasquini et al. (2004) for more details on the sample selection.

Spectra were obtained using UVES, the Ultraviolet and Visual Echelle Spectrograph (Dekker et al. 2000) fed by UT2 of the VLT. UVES is a cross-dispersed echelle spectrograph 
Table 1. Observational data of the sample stars.

\begin{tabular}{cccccc}
\hline \hline Star & $v$ & $(b-y)$ & obs. date & $t_{\exp }(\mathrm{s})$ & $S / N$ \\
\hline E3 & 12.05 & 0.38 & 10. May. 2001 & $2 \times 2100$ & 90 \\
E5 & 12.82 & 0.33 & 09. May. 2001 & $2 \times 3000$ & 90 \\
E7 & 14.17 & 0.40 & 26. Mar. 2000 & $4 \times 3600$ & 60 \\
E14 & 13.12 & 0.34 & 09. May. 2001 & $2 \times 3300$ & 95 \\
E15 & 13.52 & 0.38 & 10. Apr. 2001 & $2 \times 4500$ & 85 \\
E19 & 12.24 & 0.42 & 08. Apr. 2001 & $2 \times 2100$ & 85 \\
E34 & 13.42 & 0.36 & 11. May. 2001 & $2 \times 4500$ & 90 \\
E45 & 14.18 & 0.44 & 29. Mar. 2000 & $3 \times 3600$ & 40 \\
E56 & 12.18 & 0.38 & 08. Jun. 2001 & $2 \times 2100$ & 85 \\
E64 & 13.72 & 0.37 & 06. Jun. 2001 & $2 \times 4500$ & 100 \\
E79 & 13.55 & 0.37 & 09. Apr. 2001 & $2 \times 4500$ & 90 \\
E86 & 13.74 & 0.39 & 06. Jun. 2001 & $2 \times 4500$ & 100 \\
E95 & 11.95 & 0.52 & 08. Jun. 2001 & $2 \times 2100$ & 80 \\
E99 & 12.38 & 0.34 & 08. Jun. 2001 & $2 \times 2100$ & 90 \\
T1228 & 12.94 & 0.35 & 08. Jun. 2001 & $2 \times 3300$ & 90 \\
E25 & 12.65 & 0.37 & 07. Jun. 2001 & $2 \times 2100$ & 85 \\
T2105 & 13.95 & 0.43 & Randich et al. $(2002)$ & \\
\hline E8 & 10.67 & 0.66 & 10. May. 2001 & 1500 & 50 \\
E12 & 10.32 & 0.64 & 15. Apr. 2001 & $2 \times 1200$ & 75 \\
E60 & 10.87 & 0.67 & 09. Apr. 2001 & $2 \times 1800$ & 70 \\
E98 & 10.88 & 0.66 & 11. May. 2001 & $2 \times 1800$ & 65 \\
T812 & 11.06 & 0.67 & 07. Jun. 2001 & $2 \times 1800$ & 70 \\
\hline
\end{tabular}

Notes. The Strömgren photometry is from Meibom (2000). The $S / N$, per resolution element (4 pixels), was measured in the Be region. The five stars grouped at the end of this and the following tables are the red giants.

able to obtain spectra from the atmospheric cut-off at $300 \mathrm{~nm}$ to $\sim 1100 \mathrm{~nm}$. The reduction was conducted with the ESO UVES pipeline within MIDAS. The spectra have typical signal-to-noise $(S / N)$ between 40 and 100 and $R \sim 45000$. The log book of the observations is given in Table 1 . Stars with names starting with an "E" follow the numbering system of Eggen (1971). Stars with names starting with a "T" follow the numbering system of Anthony-Twarog et al. (1988).

The atmospheric parameters for the sample stars determined by Pasquini et al. (2004) were adopted. A first estimate of the effective temperature $\left(T_{\text {eff }}\right)$ was calculated using the calibrations of Alonso et al. (1996, 1999). A first estimate of the surface gravity was obtained using this $T_{\text {eff }}$, a mass of $1.80 M_{\odot}$ for the cluster turn-off, and the distance modulus determined by Meibom et al. (2002), $(m-M)_{0}=10.03$. Starting with these values, both the $T_{\text {eff }}$ and the microturbulence velocity $(\xi)$ were further constrained using Fe I lines, eliminating trends with the excitation potential and the equivalent widths, respectively. The ionization equilibrium, on the other hand, was not adopted to constrain the gravity. While the other parameters were being varied, gravity was also varied by at most 0.30 dex to obtain as good an equilibrium as possible without departing much from the log given by the star position at the CMD.

Using this method, Pasquini et al. (2004) had some difficulty constraining the parameters of the fast-rotating hot stars around the turn-off. The above-mentioned approach resulted in higher metallicities for these stars than the ones determined for the slow-rotating stars. This effect was likely caused by the blending of the Fe I lines with other neighboring lines due to the rotational broadening. Thus, the measured equivalent widths were greater than they should be. As in these cases the spectroscopic parameters are likely not reliable, atmospheric parameters for the fast-rotating stars were calculated adopting the photometric temperatures. The spectroscopic temperatures were adopted for

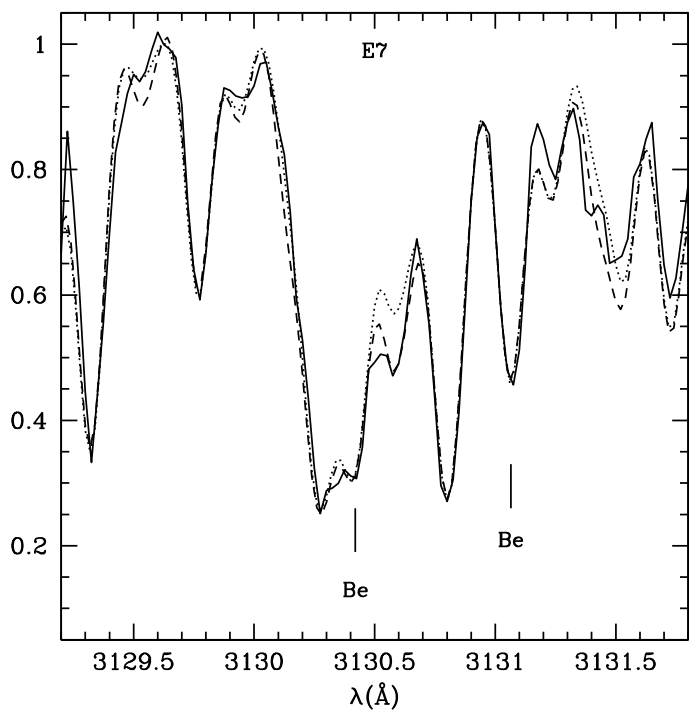

Fig. 2. Fit to the region of the Be lines in the main sequence star E7. The solid line represents the observed spectrum, the dotted line a synthetic one with solar abundance ratios, and the dashed line a synthetic spectrum with $[\mathrm{O} / \mathrm{Fe}]=+0.25$ and $[\mathrm{C} / \mathrm{Fe}]=+0.10$.

the remaining stars. The atmospheric parameters of the stars are given in Table 2 . The mean metallicity of the cluster as determined by Pasquini et al. (2004) was adopted for all the stars, $[\mathrm{Fe} / \mathrm{H}]=+0.11$.

\section{Be abundances}

\subsection{Synthetic spectra}

For late-type stars, Be abundances can only be determined using the $\mathrm{Be}$ II ${ }^{2} \mathrm{~S}-{ }^{2} \mathrm{P}_{0}$ resonance lines at $3131.065 \AA$ and $3130.420 \AA$. This near-UV region is extremely crowded with atomic and molecular lines, some of them still lacking proper identification. Thus, determination of Be abundances needs to be conducted with spectrum synthesis taking all the blending nearby features into account.

To derive the Be abundances, synthetic spectra were calculated with the codes described in Coelho et al. (2005) and the grids of model atmospheres without overshooting calculated with the ATLAS9 program (Castelli \& Kurucz 2003). These models assume local thermodynamic equilibrium, plane-parallel geometry, and hydrostatic equilibrium.

The line list is the same as used in Smiljanic et al. (2008, 2009b). In short, the molecular line list is described in Coelho et al. (2005) and the atomic line list is the one compiled by Primas et al. (1997). Log $g f$ of -0.168 and -0.468 were adopted for the Be lines at $3131.065 \AA$ and $3130.420 \AA$, respectively. The line list includes an Fe I line in $3131.043 \AA$, with $\log g f=-2.517$ and $\chi=2.85 \mathrm{eV}$, affecting the blue wing of the Be 3131 line. The parameters of this line were constrained using several stars of different parameters and metallicities (see Primas et al. 1997, for details). The proper identification of this line, however, remains controversial in the literature, and other choices have been made by different authors. As discussed in more detail by Randich et al. (2007), this choice has no significant effect on the calculation of Be abundances of stars with $T_{\text {eff }}>5400 \mathrm{~K}$. 
Table 2. Stellar parameters and abundances of $\mathrm{Li}$ and Be.

\begin{tabular}{cccccccc}
\hline \hline Star & $\begin{array}{c}T_{\text {eff }} \\
(\mathrm{K})\end{array}$ & $\begin{array}{c}\text { Spec. } \\
\text { or Phot. }\end{array}$ & $\begin{array}{c}\log g \\
(\mathrm{dex})\end{array}$ & $\begin{array}{c}\xi \\
\left(\mathrm{km} \mathrm{s}^{-1}\right)\end{array}$ & $\begin{array}{c}v \sin i \\
\left(\mathrm{~km} \mathrm{~s}^{-1}\right)\end{array}$ & $\begin{array}{c}A(\mathrm{Be}) \\
(\mathrm{dex})\end{array}$ & $\begin{array}{c}A(\mathrm{Li}) \\
(\mathrm{dex})\end{array}$ \\
\hline E3 & 6550 & Phot. & 3.90 & 2.10 & 29.9 & 1.21 & 1.64 \\
E5 & 6930 & Phot. & 4.20 & 2.00 & 23.9 & 1.36 & 3.47 \\
E7 & 6300 & Spec. & 4.30 & 1.10 & 2.1 & 1.11 & 2.83 \\
E14 & 6860 & Phot. & 4.20 & 1.90 & 34.1 & 0.76 & $\leq 2.07$ \\
E15 & 6540 & Phot. & 4.20 & 1.70 & 10.0 & 0.64 & $\leq 1.93$ \\
E19 & 6280 & Phot. & 3.90 & 2.10 & 32.1 & 0.41 & $\leq 1.66$ \\
E34 & 6640 & Phot. & 4.20 & 1.90 & 24.2 & 0.11 & $\leq 1.92$ \\
E45 & 6350 & Spec. & 4.30 & 1.10 & 4.2 & 1.08 & 2.80 \\
E56 & 6520 & Phot. & 3.90 & 2.10 & 27.8 & 1.21 & $\leq 2.16$ \\
E64 & 6650 & Spec. & 4.30 & 1.70 & 9.2 & 0.76 & 2.24 \\
E79 & 6620 & Spec. & 4.30 & 1.70 & 21.8 & 0.51 & $\leq 1.91$ \\
E86 & 6600 & Spec. & 4.30 & 1.70 & 14.0 & 0.96 & 2.20 \\
E95 & 5800 & Spec. & 3.50 & 1.70 & 12.0 & 0.61 & 2.19 \\
E99 & 6830 & Phot. & 4.00 & 2.00 & 28.1 & 1.21 & $\leq 2.38$ \\
T1228 & 6770 & Phot. & 4.20 & 1.70 & 5.7 & 1.21 & 3.18 \\
E25 & 6680 & Phot. & 4.00 & 2.00 & 21.3 & 1.11 & 3.29 \\
T2105 & 6110 & Phot. & 4.44 & 1.10 & 8.0 & 1.11 & 2.82 \\
\hline E8 & 4900 & Spec. & 2.70 & 1.30 & 0.1 & - & $\leq 0.09$ \\
E12 & 5000 & Spec. & 2.70 & 1.50 & 1.1 & $\leq-0.70$ & $\leq 0.35$ \\
E60 & 4900 & Spec. & 2.90 & 1.40 & 1.9 & $\leq-0.70$ & 0.42 \\
E98 & 4900 & Spec. & 3.00 & 1.40 & 1.0 & $\leq-0.50$ & 0.72 \\
T812 & 5000 & Spec. & 3.00 & 1.60 & 0.5 & $\leq-0.70$ & $\leq 0.38$ \\
\hline
\end{tabular}

\subsection{The Sun and the slow-rotating stars}

That some of the sample stars are fast rotators adds further difficulty to the analysis of the Be spectral region. Thus, we conducted a careful analysis, first modeling the slow-rotating stars and conducting a few tests to make certain any possible systematic effect affecting the modeling of the fast-rotating stars would be under control.

As the first step, with the line list described above, we fitted the Be lines in the solar $\mathrm{UVES}^{1}$ spectrum, adopting the parameters: $T_{\text {eff }}=5777 \mathrm{~K}, \log g=4.44$, and $\xi=1.00 \mathrm{~km} \mathrm{~s}^{-1}$. An abundance of $A(\mathrm{Be})=1.10$ was obtained. This abundance is in excellent agreement with the one found by Chmielewski et al. (1975), $A(\mathrm{Be})=1.15$, usually adopted as the reference photospheric solar abundance.

The low photospheric solar Li abundance, depleted by a factor of $\sim 160$ with respect to the meteoritic value, has been known for a long time (see e.g. Müller et al. 1975). The photospheric Be abundance and its difference with respect to the meteoritic one have instead been a matter of debate. All analyses of the solar photospheric abundance obtain $\log (\mathrm{Be} / \mathrm{H}) \sim 1.15-1.25$ (Chmielewski et al. 1975; Garcia Lopez et al. 1995; Randich et al. 2002), a factor of 2 less than what is measured in meteorites, $\log (\mathrm{Be} / \mathrm{H})=1.41($ Lodders 2003). This issue now seems settled to a large extent; the measured photospheric value is lower than the real one due to our inability of properly accounting all continuum opacity sources in the region around $313 \mathrm{~nm}$, where the two Be II resonance lines are located, when calculating model atmospheres (Balachandran \& Bell 1998; Asplund 2004).

The second step was to fit the two main sequence slowrotating stars E7 and E45. The cluster was found to have a solar abundance pattern by Pasquini et al. (2004). Thus, the solar

\footnotetext{
1 The spectrum is available for download at the ESO website: www. eso.org/observing/dfo/quality/UVES/pipeline/solar_ spectrum.html
}

abundance ratios ${ }^{2}$ were adopted when calculating a first set of synthetic spectra. However, as shown in Fig. 2, when adopting a solar abundance pattern, an important molecular feature near the $3130 \AA$ Be line is not properly fitted. A better fit is obtained when $[\mathrm{O} / \mathrm{Fe}]$ is increased by $+0.25 \mathrm{dex}$ and $[\mathrm{C} / \mathrm{Fe}]$ by $+0.10 \mathrm{dex}$, in both E7 and E45. These apparent overabundances are likely not to be real but caused by shortcomings in the analysis, such as the neglect of effects caused by departures from the local thermodynamic equilibrium and/or by problems with the molecular and atomic data in this region.

Nevertheless, it is important to notice that properly fitting the neighboring molecular features in the slow-rotating stars is an important step toward analyzing the fast-rotating ones. In the last case, all these lines are blended into a single feature. As all the stars in the cluster are expected to have the same chemical composition (except for the giants), one may expect that the enhanced abundances of $\mathrm{C}$ and $\mathrm{O}$ are also needed to properly fit the spectra of the fast-rotating stars. We stress that this increase in the abundances of $\mathrm{C}$ and $\mathrm{O}$ has no effect on the calculated $\mathrm{Be}$ abundances of the slow-rotating stars. Thus, when fitting the fast rotators, $\mathrm{C}$ and $\mathrm{O}$ abundances were kept with these increased values, while the other elements were kept with a solar ratio. Only the $\mathrm{Be}$ abundance was varied from star to star. The derived Be abundances are listed in Table 2.

\subsection{The fast-rotating stars}

Before fitting the true fast-rotating stars, we conducted a test to better understand the effects of the broadening in the derived abundances. We artificially broadened the spectra of the two slow-rotating stars, E7 and E45, by convolving them with

\footnotetext{
2 The abundances recommended by Grevesse \& Sauval (1998) were adopted for all elements except for oxygen, for which we adopted the abundance suitable for the 1D atmospheric models recommended by Allende Prieto et al. (2001), $A(\mathrm{O})=8.77$, and for Be for which the abundance derived above from the solar spectrum was adopted.
} 


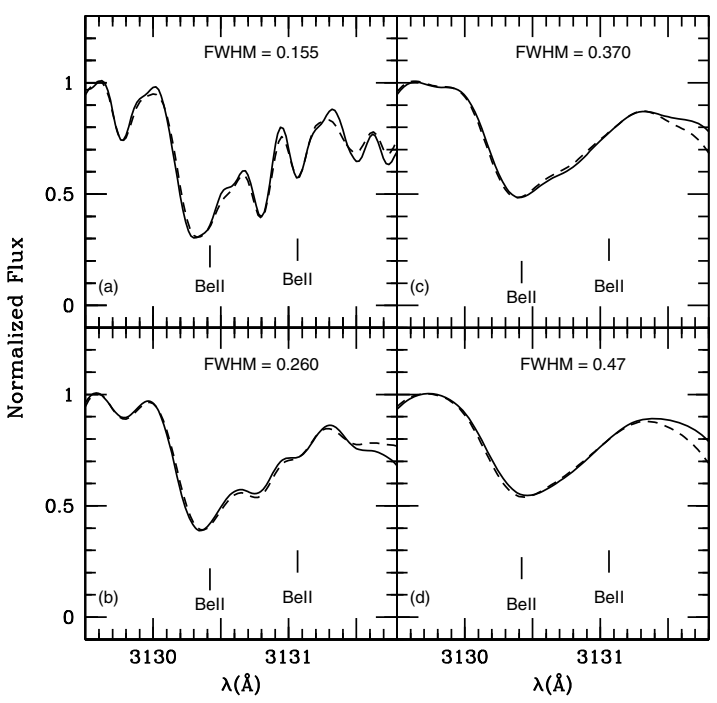

Fig. 3. Fit to the region of the Be lines in the main sequence star E7. The four panels show a sequence of larger broadening applied to the observed spectrum. In all panels the best-fitting synthetic spectrum has $A(\mathrm{Be})=1.16 \mathrm{dex}$.

Gaussian functions. In Fig. 3 we show the spectrum of the star E7 broadened with four different Gaussian functions. The Be abundance was then calculated for these broadened spectra.

In the four cases shown in Fig. 3, an abundance of $A(\mathrm{Be})=$ 1.16 dex was derived for $\mathrm{E} 7$, while $A(\mathrm{Be})=1.11$ dex was derived in the original unbroadened spectrum. Applying similar broadening to $\mathrm{E} 45$, we obtain $A(\mathrm{Be})=1.15$ for all the broadened cases, while $A(\mathrm{Be})=1.08 \mathrm{dex}$ was obtained for the original observed spectrum. Thus, we conclude that the abundances in a broadened spectrum are slightly overestimated. However, this small amount is well within the uncertainties (see below). Therefore, we are confident that it is possible to derive meaningful abundances for the fast-rotating stars with our method of analysis.

Beryllium abundances were then derived for all the fastrotating main-sequence and turn-off stars. The stars were found to have a broad range in abundances, from 1.36 dex (E5) to 0.11 dex (E34). In Fig. 4 we plot the spectra of these two stars, which have similar $v \sin i$, to show that the difference in Be abundance obtained in the analysis is real. In the lower panel of this same figure we show the result of the division of these spectra. The structures left in the division have central wavelengths of 3130.41 and $3131.03 \AA$, arguing that the difference is really caused by the different Be abundances. Similarly, we compare in Fig. 5 the observed spectrum of star E34 and the spectrum of E7 artificially broadened to a $v \sin i$ similar to that of E34. It is clear from this figure that star E34 has a smaller Be abundance than E7. This comparison again shows that the difference in Be abundances detected in the stars is real.

Finally, we point out that Be was detected in all the sample of main sequence and turn-off stars. Most of the stars show signs of Be depletion, i.e., Be lower than the meteoritic value, but still have a detectable amount of Be in their photospheres. In Fig. 6 we show the observed spectra of E19 and E34, the two stars with smaller Be content. Also shown are a best-fit synthetic spectrum together with a spectrum calculated with no Be at all. As clearly seen, the spectra lacking Be show a marked difference when compared to the observed ones. This comparison argues that our Be abundances are actual detections and not upper-limits. The derived Be abundances are listed in Table 2.

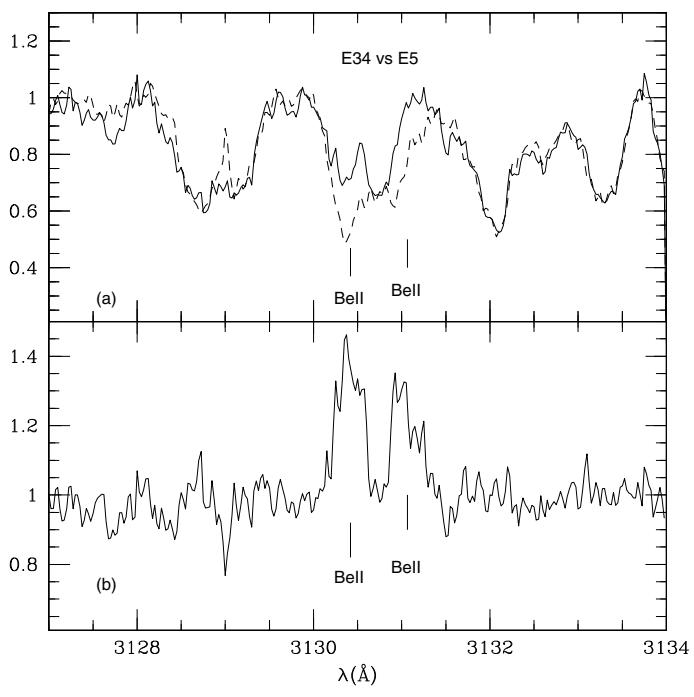

Fig. 4. a) Comparison between the observed spectra of stars E5 (dashed line) and E34 (solid line). The stars have similar rotational velocity and different Be abundances. b) The result of the division of the two spectra in the upper panel. The two residual features are located at 3130.41 and $3131.03 \AA$ A. These wavelengths are compatible with the difference being caused by different Be abundances.

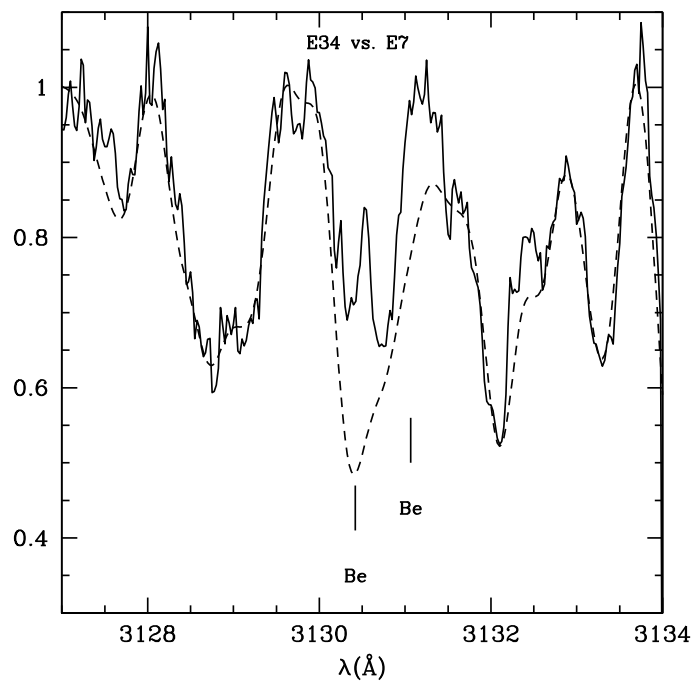

Fig. 5. Comparison between the observed spectrum of star E34 (solid line) and the spectrum of star E7 (dashed line) artificially broadened to a $v \sin i$ similar to the one of E34. It is clear the lower Be abundance of star E34 and that a higher abundance could be easily detected in our analysis.

\subsection{The red giants}

Fitting synthetic spectra in the Be region for the red giants proved to be even more challenging than fitting the fast-rotating stars. From this exercise it became clear that the line list used to fit the main sequence and turn-off stars is not fully adequate for red giants. Problems with the wavelengths and depths of the lines become clear. More work on the transitions affecting the near UV region is required.

In particular, the Fe I line adopted as the blend affecting the $3131 \AA$ Be line appears to be too strong here and with a wrong wavelength when compared to the observed spectra (see Fig. 7). For the dwarf stars, as discussed before, this blend has no significant effect. For metal-poor stars, such as the subgiants 


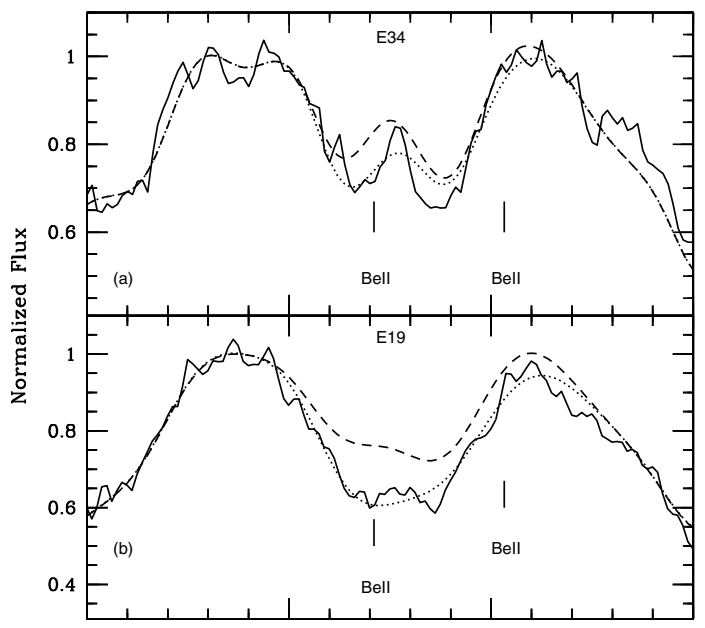

Fig. 6. a) Comparison between the observed spectrum of star E34 (solid line), the best synthetic fit (dotted line), and a synthetic spectrum calculated without $\mathrm{Be}$ (dashed line). b) Comparison between the observed spectrum of star E19, the best synthetic fit, and a synthetic spectrum calculated without Be. These two comparisons argue for both stars having detectable amounts of Be.

analyzed by García Pérez \& Primas (2006), the blending feature is also not significant. For our metal-rich cool giants, however, it prevents reliable abundances from being derived from this $\mathrm{Be}$ line. In this case, we relied solely on the $3130.4 \AA$ feature.

The $3130.4 \AA$ line, however, is very weak (indicating that $\mathrm{Be}$ is strongly depleted or even absent in giants) and strongly blended. In Fig. 7 an example of fitting the Be region in a red giant is given. The Be line only affects the wing of a very strong and heavily blended feature. The best fit is found when the wing of this feature in the synthetic spectrum is brought to the level of the wing in the observed spectrum. The figure suggests that an abundance cannot be derived in this case with an uncertainty better than \pm 0.20 dex.

One should also note that the abundances in this case are strongly dependent on broadening, blending features (their inclusion and also on the right abundance for the given element), and on the determination of the continuum. Thus, one should be very careful when interpreting the abundances derived for the red giants. In this case we consider that only an upper limit on the $\mathrm{Be}$ abundance can be determined. In addition, the difficulties discussed above mean that one should be very careful not to push the comparison between dwarfs and giants too far. The Be upper limits for the red giants are listed in Table 2.

Of all these issues, the level of the continuum is of particular concern. Different solutions could bring the wing of the line up or down, changing the best fit. Finding the correct level of the continuum in the near UV region is at best a very difficult task. However, by adopting very similar solutions for all the stars that have very similar atmospheric parameters, we believe that at least a reliable differential scale can be obtained.

Regarding the abundances of other elements, it is expected that the first dredge-up would change $\mathrm{C}$ and $\mathrm{N}$ abundances of the stars on the red giant branch (RGB). This near UV region is affected by many $\mathrm{CN}, \mathrm{NH}$, and $\mathrm{CH}$ lines, so this change in abundances could be an important issue. We calculated a synthetic spectrum for star E60 by adopting typical post dredge-up values for these elements, $[\mathrm{C} / \mathrm{Fe}]=-0.15$ and $[\mathrm{N} / \mathrm{Fe}]=+0.40$ (see e.g. Smiljanic et al. 2009a). A comparison with the best fit obtained using unaltered abundances showed only very weak effects, well within the \pm 0.20 uncertainty of the fit.

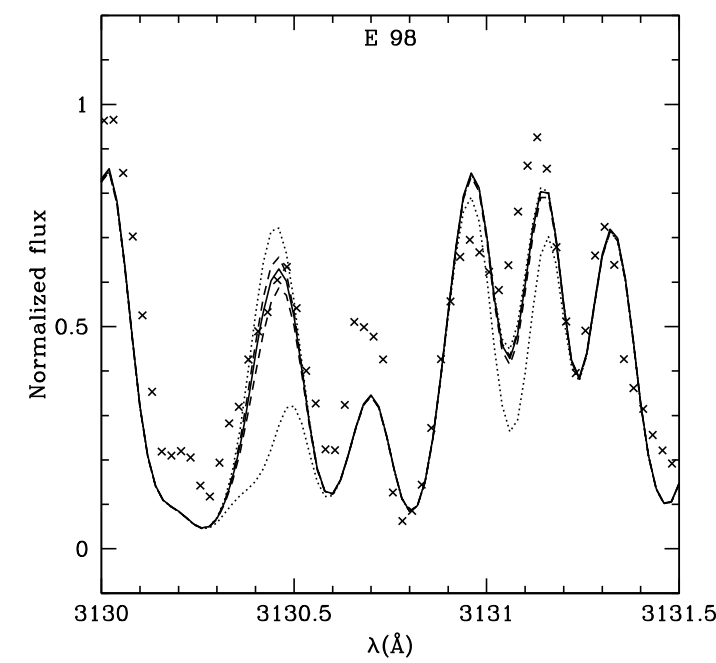

Fig. 7. Fitting the Be lines in the red giant E98. The crosses represent the observed spectrum, the solid line the best fit, the dashed lines represent variations of \pm 0.20 dex from the best fit, and the dotted lines represent a spectrum without $\mathrm{Be}$ and one with $A(\mathrm{Be})=1.21$.

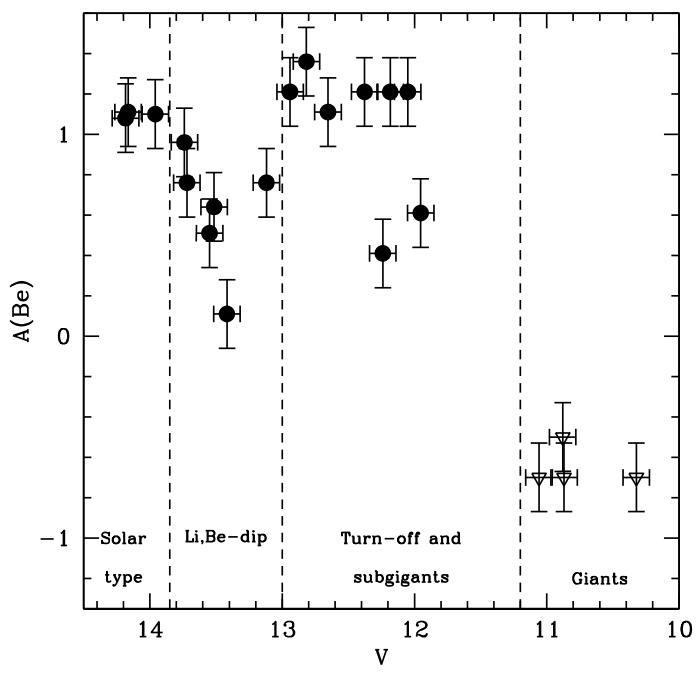

Fig. 8. The Be abundances of the sample stars, calculated in this work, as a function of the $V$ magnitude. Abundance determinations are shown as solid circles and upper limits as open triangles. The Be dip is clearly seen.

The Be abundances of all sample stars are shown as a function of the $V$ magnitude in Fig. 8. For comparison, we also show a plot of the Li abundances determined by Pasquini et al. (2004) as a function of $V$ in Fig. 9. The evolutionary stages of the stars are indicated in the figures. It is clearly seen that the stars of the Li dip also define a clear Be dip.

\subsection{Uncertainties and comparison with the literature}

The determination of Be abundances is affected by the uncertainties of the atmospheric parameters and by the uncertainty in the determination of the pseudo-continuum during the spectrum fitting. To conduct the calculations of the errors, we adopted the same uncertainties of the atmospheric parameters determined by Pasquini et al. (2004), $\sigma_{T_{\text {eff }}}= \pm 100 \mathrm{~K}, \sigma_{\log g}= \pm 0.30 \mathrm{dex}, \sigma_{\xi}=$ $\pm 0.30 \mathrm{~km} \mathrm{~s}^{-1}$, and $\sigma_{[\mathrm{Fe} / \mathrm{H}]}= \pm 0.10 \mathrm{dex}$.

To estimate the effect of the atmospheric parameters, we can change each one by its own error, keeping the other ones with the 
Table 3. The uncertainties of the Be abundance introduced by the uncertainties of the atmospheric parameters and by uncertainty of the fitting itself related to the level of the continuum and the $S / N$.

\begin{tabular}{ccccccc}
\hline \hline Star & $\sigma_{T_{\text {eff }}}$ & $\sigma_{\log g}$ & $\sigma_{\xi}$ & $\sigma_{[\mathrm{Fe} / \mathrm{H}]}$ & $\sigma_{\text {fitt. }}$ & $\sigma_{\text {total }}$ \\
\hline E 7 & \pm 0.03 & \pm 0.10 & \pm 0.00 & \pm 0.00 & \pm 0.05 & \pm 0.12 \\
E 86 & \pm 0.05 & \pm 0.10 & \pm 0.05 & \pm 0.05 & \pm 0.10 & \pm 0.17 \\
E 60 & \pm 0.15 & \pm 0.05 & \pm 0.03 & \pm 0.05 & \pm 0.20 & \pm 0.26 \\
\hline
\end{tabular}

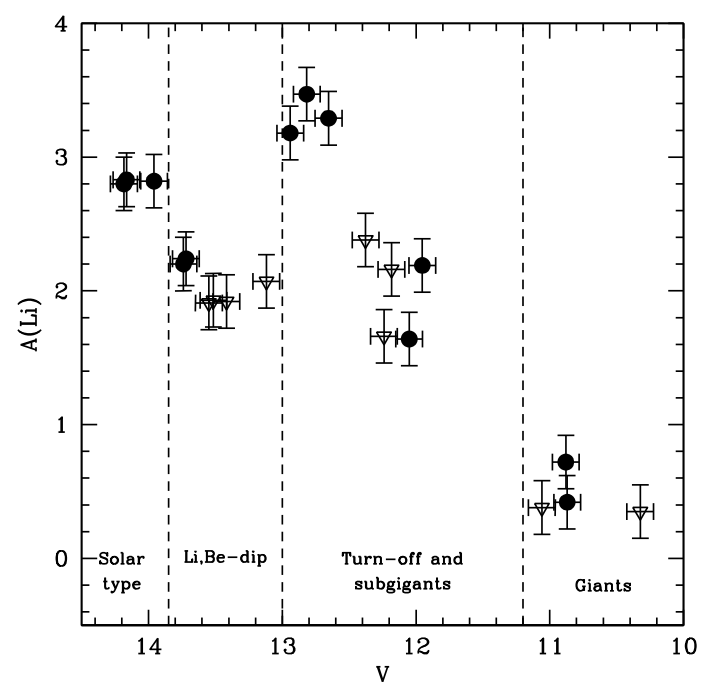

Fig. 9. The Li abundances of the sample stars, calculated in Pasquini et al. (2004), as a function of the $V$ magnitude. Abundance determinations are shown as solid circles and upper limits as open triangles.

original adopted values, and recalculate the abundances. Thus, we measure what effect the variation of one parameter has on the abundances. We assume the uncertainties introduced by each parameter to be independent of the other ones. The uncertainty due to the continuum was determined by estimating the sensitivity of the derived Be abundance on the adopted continuum level. This uncertainty is mostly related to the $S / N$ of the spectrum. These effects are listed in Table 3. The calculations were done for three representative stars, E7 as an example of slow-rotating star, E86 as an example of a fast-rotating star, and E60 as an example of a giant star. The $\mathrm{Li}$ and $\mathrm{Be}$ abundances derived for the star E5, which seem to be slightly above the solar values (see Table 2), seem to suggest that the uncertainty on its temperature is slightly higher than for the other stars. For these hot stars, the effective temperature is also important for determining $\mathrm{Be}$ abundances. We thus believe that its $\mathrm{Li}$ and $\mathrm{Be}$ abundances are slightly overestimated and should be interpreted with care.

As mentioned in Sect. 2, Randich et al. (2002) has analyzed three main sequence stars of IC 4651 and determined their Be abundances. Two of their stars, E7 and E45, are also analyzed here. The atmospheric parameters derived by Randich et al. (2002) are different from the ones adopted here. Randich et al. (2002) calculated temperatures of 6061 and $6016 \mathrm{~K}$, while we adopted 6300 and $6350 \mathrm{~K}$ for stars E7 and E45, respectively. A gravity of $\log g=4.44$ was adopted by Randich et al. (2002) for all stars they analyzed, while a value of $\log g=4.30$ was calculated for both E7 and E45 in Pasquini et al. (2004) and adopted here. In spite of these differences, the Be abundances of the two stars as derived by Randich et al. (2002) agree with ours within the uncertainties; that is, Randich et al. (2002) derived
$A(\mathrm{Be})=1.11$ and 1.16 for stars E7 and E45, while we obtained $A(\mathrm{Be})=1.11$ and 1.08 , respectively.

\section{Comparison with model predictions for rotating stars}

\subsection{Input physics of the hydrodynamical stellar models}

\subsubsection{Hot side of the Li dip}

In Pasquini et al. (2004) the $\mathrm{Li}$ abundance patterns in IC 4651 were compared with model predictions for rotating stars by Charbonnel \& Talon (1999) and Palacios et al. (2003). These models include atomic diffusion, as well as the transport of angular momentum and chemicals by meridional circulation and shear turbulence following the formalism by Zahn (1992), Talon \& Zahn (1997), and Maeder \& Zahn (1998) (for more details and references see the original papers as well as Decressin et al. 2009). They are adequate for stars that lie on or originate in the hot side of the dip and inside which angular momentum transport by internal gravity waves emitted by convective envelopes is inefficient (Talon \& Charbonnel 2003). These models were found to explain the corresponding $\mathrm{Li}$ data for both main sequence and giant stars in the intermediate-age IC 4651 (see Pasquini et al. 2004), as they do for younger clusters like the Hyades, Coma Berenices, and Praesepe (see e.g. Charbonnel \& Talon 2008).

Meanwhile new stellar models within the same mass range (i.e., for stars more massive than $1.2 M_{\odot}$ ) were computed for other purposes with the stellar evolution code STAREVOL V3.1 (Charbonnel \& Lagarde, in preparation) using the same prescriptions for the transport of angular momentum and chemicals by meridional circulation and shear turbulence as in the abovementioned papers. Atomic diffusion is also included in the form of gravitational settling, as well as that related to thermal gradients, using the formulation by Paquette et al. (1986). As far as the input physics is concerned, the differences with the models by Charbonnel \& Talon (1999) and Palacios et al. (2003) are the following: (1) the reference solar composition is the one derived by Asplund et al. (2006), except for Ne for which the recommendations by Cunha et al. (2006) are followed; (2) new OPAL opacity tables are used according to the chemical mixture; (3) thermohaline mixing is accounted for following Charbonnel \& Zahn (2007), a process that only becomes efficient when the stars reach the so-called luminosity bump on the RGB (see Sect. 4.3).

Results of these recent computations for initial stellar masses of $1.3,1.35,1.4,1.5,1.8$, and $2 M_{\odot}$ for $\mathrm{Z}=0.014$ are presented. In view of the remaining uncertainty on the age of IC 4651 , we provide in Table 4 relevant model characteristics at 1.2 and 1.7 Gyr for the stars that are still on the main sequence at that time. In all cases various initial rotation velocities on the zero-age main sequence were considered. (The 1.3, 1.35, 1.4, and $1.5 M_{\odot}$ models are computed for $V_{\text {ZAMS }}=50,80$, and $110 \mathrm{~km} \mathrm{~s}^{-1}$; the $1.8 M_{\odot}$ models are computed for $V_{\text {ZAMS }}=80$, 110 , and $180 \mathrm{~km} \mathrm{~s}^{-1}$, and the $2 M_{\odot}$ models for $V_{\mathrm{ZAMS}}=110$, 180 , and $250 \mathrm{~km} \mathrm{~s}^{-1}$.) The efficiency of magnetic braking applied to stars arriving on the main sequence is determined according to the work of Gaigé (1993) following the braking law by Kawaler (1988) (for more details see Palacios et al. 2003). In practice, magnetic braking only has to be applied to the 1.3, 1.35, and $1.4 M_{\odot}$ stars that have thicker convective envelopes than more massive stars. As in previous works, the aim was to reproduce the rotation velocity distribution observed at the age of the Hyades. Theoretical rotation velocities at the age of IC 4651 are given in Table 4 and span the velocity range of our sample 
Table 4. Rotation velocity, effective temperature, and surface abundances of $\mathrm{Li}$ and Be predicted for the stellar models that are still on the main sequence at the age of IC 4651

\begin{tabular}{cccccccccc}
\hline \hline $\begin{array}{c}M \\
\left(M_{\odot}\right)\end{array}$ & $\begin{array}{c}V_{\text {ZAMS }} \\
\left(\mathrm{km} \mathrm{s}^{-1}\right)\end{array}$ & \multicolumn{2}{c}{$V_{\text {Age }}$} & \multicolumn{2}{c}{$T_{\text {eff }}$} & \multicolumn{2}{c}{$A(\mathrm{Li})$} & \multicolumn{2}{c}{$A(\mathrm{Be})$} \\
& & $\left.1.2 \mathrm{Gyr} \mathrm{km}^{-1}\right)$ & $1.7 \mathrm{Gyr}$ & $1.2 \mathrm{Gyr}$ & $1.7 \mathrm{Gyr}$ & $1.2 \mathrm{Gyr}$ & $1.7 \mathrm{Gyr}$ & $1.2 \mathrm{Gyr}$ & $1.7 \mathrm{Gyr}$ \\
\hline \hline 1.3 & 50 & 19.4 & 16.9 & 6532 & 6507 & 2.05 & 1.51 & 1.02 & 0.77 \\
& 80 & 28.3 & 24.4 & 6535 & 6528 & 1.24 & 0.37 & 0.62 & 0.22 \\
& 110 & 36.2 & 30.9 & 6520 & 6483 & 0.47 & -0.59 & 0.2 & -0.29 \\
\hline 1.35 & 50 & 30.3 & 27.6 & 6687 & 6663 & 2.69 & 2.33 & 1.26 & 1.10 \\
& 80 & 28.6 & 24.5 & 6683 & 6670 & 1.16 & 0.24 & 0.57 & 0.15 \\
& 110 & 36.6 & 31.5 & 6685 & 6676 & 0.43 & -0.70 & 0.20 & -0.35 \\
\hline 1.4 & 50 & 42.7 & 41.4 & 6834 & 6762 & 3.24 & 3.22 & 1.41 & 1.41 \\
& 80 & 67.8 & 67.8 & 6824 & 6764 & 3.20 & 3.10 & 1.41 & 1.39 \\
& 110 & 96 & 95.2 & 6826 & 6781 & 3.12 & 2.94 & 1.39 & 1.33 \\
\hline 1.5 & 50 & 40.5 & 37.5 & 7094 & 6857 & 3.23 & 3.18 & 1.41 & 1.40 \\
& 80 & 66.5 & 62.7 & 7174 & 6948 & 3.13 & 2.94 & 1.40 & 1.34 \\
& 110 & 92.2 & 87.8 & 7136 & 6900 & 2.97 & 2.66 & 1.35 & 1.21 \\
\hline
\end{tabular}

Notes. Models by Charbonnel \& Lagarde (in preparation). See text for more details.

stars. In all cases the surface rotation velocity also changes because of secular evolution, and it is reduced to only a few $\mathrm{km} \mathrm{s}^{-1}$ when the models are on the subgiant branch due to the radius expansion.

\subsubsection{Cool side of the Li dip}

For less massive main-sequence stars lying on the cool side of the $\mathrm{Li}$ dip that have deeper convective envelopes, internal gravity waves are efficiently generated and participate in the extraction of internal angular momentum, along with meridional circulation and shear turbulence (Talon \& Charbonnel 2003). In this case, the appropriate models are those by Talon \& Charbonnel (2005) and Charbonnel \& Talon (2005), which take all these effects into account. Unfortunately the $1.0 M_{\odot}$ models of Charbonnel \& Talon (2005) have an effective temperature at the age of the IC 4651 (i.e., 5597 K), lower than our cooler main sequence stars, and they cannot be used for comparison with the present sample. Obviously abundance determinations for such relatively cool objects would be extremely valuable for further tests of the models. Only the $1.2 M_{\odot}$ model computed by Talon \& Charbonnel (2005) is available for comparison purposes (see Sect. 4.2). It has an initial rotation velocity on the zero age main sequence of $50 \mathrm{~km} \mathrm{~s}^{-1}$, and surface spin-down follows Kawaler (1988) as explained in Sect. 4.1.1.

\subsubsection{Initial values for $\mathrm{Li}$ and $\mathrm{Be}$}

In the following we compare the predictions of the hydrodynamical models described above with the observations of both $\mathrm{Li}$ and Be in IC 4651. Results are presented separately for the main sequence and turnoff stars (Sect. 4.2) and for more evolved stars (Sect. 4.3). In the following figures, we use the $V$ magnitudes to divide the stars according to their evolutionary stages; stars fainter than $V=12.6$ are at the main sequence, stars between 12.6 and 11.8 are at the upper turn-off or at the subgiant branch, and stars brighter than 11.8 are giants.

For the initial values of $A(\mathrm{Li})$ and $A(\mathrm{Be})$ used in our computations we chose the meteoritic abundances, i.e., 3.25 (Asplund et al. 2006) and 1.41 (Lodders 2003), respectively. This Li value is actually lower than the highest $\mathrm{Li}$ abundance determined among our sample stars, which induces an artificial shift within the comparison between predictions and data. For this reason we

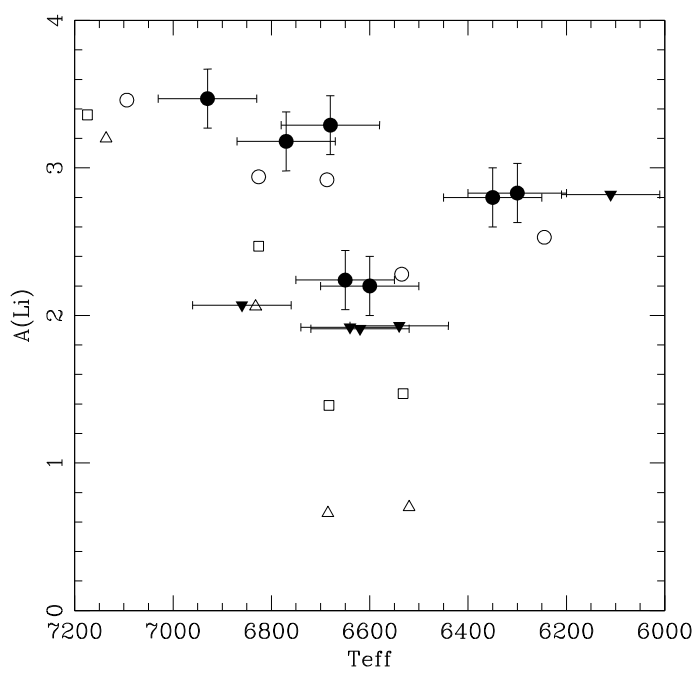

Fig. 10. Li abundances in IC 4651 main sequence and turnoff stars fainter than $V \sim 12.6$ (black points and triangles for actual determinations and upper limits, respectively). Open circles, squares, and triangles show model predictions for initial rotation velocities of 50, 80, and $110 \mathrm{~km} \mathrm{~s}^{-1}$, respectively. For comparison purposes the theoretical $\mathrm{Li}$ points are increased by 0.16 dex to account for the difference between the meteoritic value and the highest $\mathrm{Li}$ abundance determined in our sample stars. See text for more details.

increase, within the figures, the theoretical Li points by 0.16 dex. This corresponds to assuming that the highest Li abundance derived among our sample stars $(\mathrm{E} 5, A(\mathrm{Li})=3.47 \mathrm{dex})$ is the initial abundance of the sample stars.

\subsection{Comparisons with observed features on the main sequence}

Figures 10 and 11 compare the predictions of the stellar models described in Sect. 4.1 to the $\mathrm{Li}$ and Be observed abundances of the main-sequence and turn-off stars of our IC 4651 sample as a function of the effective temperature. The models provide an estimate of the expected dispersion in surface abundances due to a dispersion in initial rotation velocities. On the cool side of the dip, only one theoretical point is available in the temperature range considered in the present study $\left(1.2 M_{\odot}\right.$, $V_{\text {ZAMS }}=50 \mathrm{~km} \mathrm{~s}^{-1}$ model of Talon \& Charbonnel 2005). 


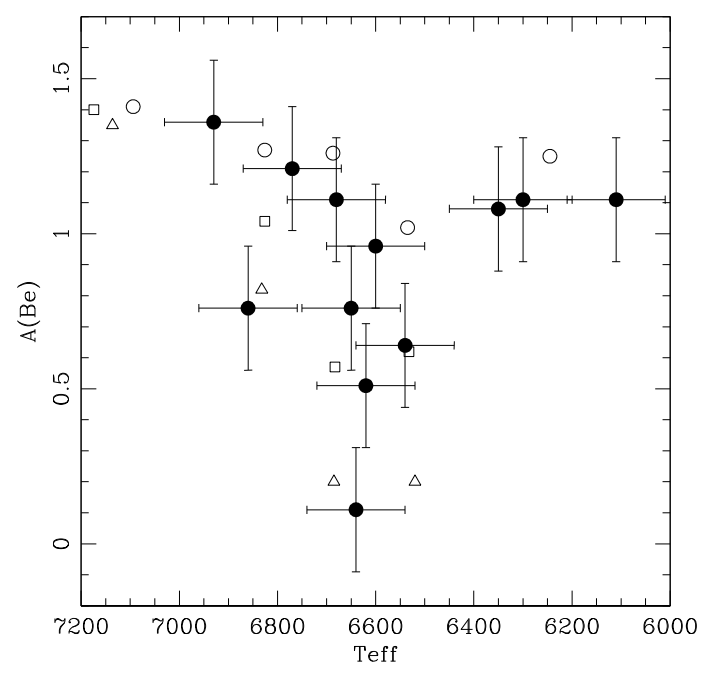

Fig. 11. Same as Fig.10 for observed and theoretical Be abundances.

It is possible to note on these graphs (see also Fig. 12) that the observational behavior of the Be abundances follows that of the Li abundances closely. The Li dip is also a Be dip, confirming previous literature results (Boesgaard \& King 2002; Boesgaard et al. 2004a). In a narrow range in effective temperature centered on $\sim 6650 \mathrm{~K}$, stars present both an $\mathrm{Li}$ and a Be depletion. Important is that $\mathrm{Li}$, which is more fragile (it burns indeed through proton-captures at $\sim 2.5 \times 10^{6} \mathrm{~K}$ compared to $\sim 3.5 \times 10^{6} \mathrm{~K}$ for $\mathrm{Be}$ ), is more strongly depleted within the dip. In most of the stars lying in this region, only upper limits could be derived for $\mathrm{Li}$, while Be is always detected.

The predictions of the models described in Sect. 4.1 successfully account for the Li patterns as a function of effective temperature, namely, the presence of a large fraction of stars with Li upper limits between $\sim 6500$ and $6800 \mathrm{~K}$ (i.e., within the dip) and the higher Li values on both the cool and hot sides of the dip (see Fig. 10). They also reproduce the Be behavior remarkably well in the whole effective temperature range considered (see Fig. 11). The success of the models is confirmed when plotting Be versus $\mathrm{Li}$ as shown in Fig. 12. As noted in Sect. 4.1.3, the theoretical $\mathrm{Li}$ values have been shifted by 0.16 dex to account for the difference between the meteoritic $\mathrm{Li}$ and the highest $\mathrm{Li}$ abundance determined in our sample stars; however, what really matters for constraining the models is the slope of the trend between $\mathrm{Li}$ and Be. As can be seen in Fig. 12, the theoretical and observational slopes are found to agree very nicely. Linear fits of the observational values, accounting for errors in both axes, have slopes of $+0.58 \pm 0.09$, if all detections and upper limits are considered, of $+0.45 \pm 0.05$ if we only exclude star E34 (the one with the smaller Be abundance), and of $+0.35 \pm 0.08$ if we consider only the detections. For the theoretical values we calculate a slope of +0.45 considering all points. If we restrict the theoretical values to the ones with $A(\mathrm{Li})>2.0$, which is the range where the observational detections are, a slope of +0.33 is obtained. These values agree very well with the observed ones. Similar correlated depletions of $\mathrm{Li}$ and $\mathrm{Be}$ have also been observed in field and cluster stars by Deliyannis et al. (1998), Boesgaard et al. (2004b), and Randich et al. (2007).

On the cool side of the dip (i.e., below $\sim 6400 \mathrm{~K}$ ), we find no Be dispersion thus confirming previous results by Boesgaard et al. (2004b) and Randich et al. (2002, 2007). This provides further constraints to models of rotating low-mass (i.e., below $\sim 1.25 M_{\odot}$ ) stars including the transport of angular momentum by gravity waves that should assume a range in initial

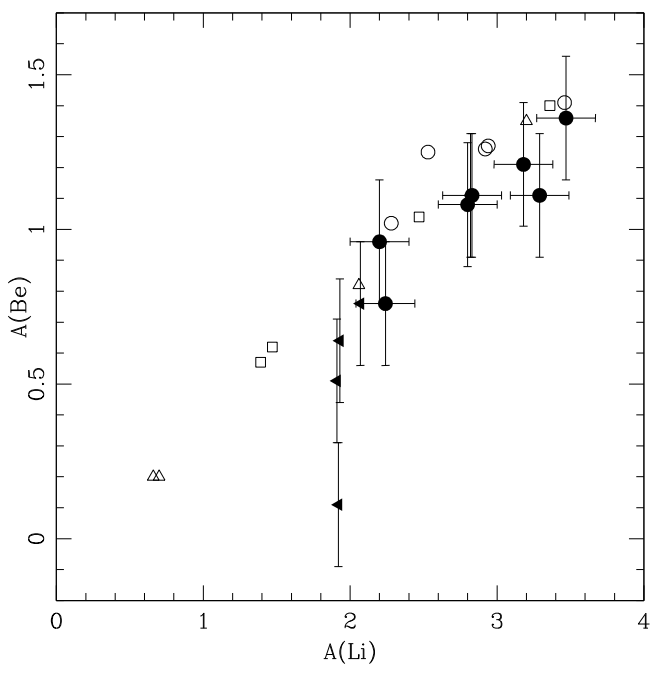

Fig. 12. Li and Be abundances in IC 4651 main sequence and turnoff stars fainter than $V \sim 12.6$ (black points and triangles for Li actual determinations and upper limits, respectively). Open circles, squares, and triangles show model predictions for initial rotation velocities of 50, 80, and $110 \mathrm{~km} \mathrm{~s}^{-1}$, respectively. For comparison purposes the theoretical Li points are increased by 0.16 dex to account for the difference between the meteoritic value and the highest $\mathrm{Li}$ abundance determined in our sample stars. See text for more details.

rotation velocities (Charbonnel et al. in preparation) $)^{3}$. However, we can already note that models of solar-mass stars computed with a large range of initial angular momentum ( $V_{\text {ini }}$ from 15 to $110 \mathrm{~km} \mathrm{~s}^{-1}$ ) and including the effects of internal gravity waves predict very small Li dispersion at the age of IC 4651 (see Charbonnel \& Talon 2005), and thus even more modest Be dispersion. This is caused by internal gravity waves that dominate the transport of angular momentum in stars lying on the red side of the dip and enforce quasi solid-body rotation within the stellar interior. As a result, the surface Li and Be depletions are expected to almost be independent of the initial angular momentum distribution (i.e., of the initial rotation velocity), implying very low surface abundance variations from star to star. On the other hand, in more massive (i.e., higher than $\sim 1.25 M_{\odot}$ ) stars the efficiency of internal gravity waves dramatically drops and internal differential rotation is expected to be maintained along the stellar life under the effects of meridional circulation and turbulence. Consistently, variations in the initial angular momentum from star to star lead to more $\mathrm{Li}$ and Be dispersion, as required by the observations.

\subsection{Comparisons with features observed in subgiant and giant stars}

Let us now focus on the post-main-sequence evolution. Figures 13 and 14 compare the $\mathrm{Li}$ and $\mathrm{Be}$ abundances of our

${ }^{3}$ Randich et al. (2002) compared the Li and Be abundances determined in early G-type stars in old clusters, among which IC 4651 with predictions of models including gravity waves by Montalbán \& Schatzman (2000). In these models, rotation-induced processes are ignored, and waves are invoked as the only source of mixing for chemicals in the stellar radiative zone. This is very different from the view proposed by Charbonnel \& Talon. In this case indeed gravity waves transport angular momentum, together with meridional circulation and turbulence, but the transport of chemicals is essentially caused by rotation-induced mixing. See the original papers for more detailed information. 


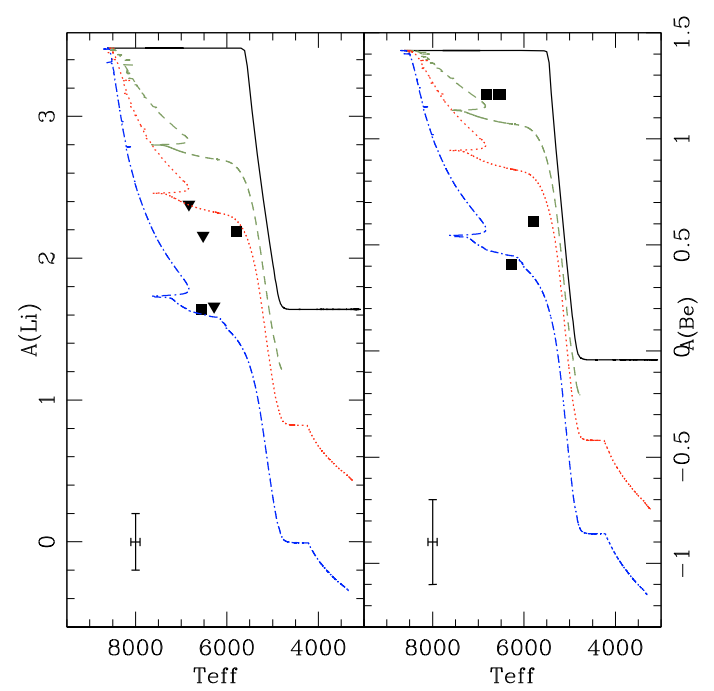

Fig. 13. Li and Be abundances in IC 4651 subgiant stars with $T_{\text {eff }}$ between $\sim 6830$ and $5800 \mathrm{~K}$ and $\mathrm{V}$ between 12.6 and 11.8. Theoretical predictions for the surface $\mathrm{Li}$ and $\mathrm{Be}$ evolution are shown for the $1.8 M_{\odot}$ star (see text for more details). The solid line is for the classical case, while the other lines present the results for the rotating models computed with different initial rotation velocities $\left(80,110\right.$, and $180 \mathrm{~km} \mathrm{~s}^{-1}$, respectively, for dashed, dotted, and dot-dashed lines). The Li tracks are shifted by 0.16 dex as explained in the text.

subgiant and giant sample stars, as a function of the effective temperature (which now depicts evolutionary stage), with predictions of our most massive models computed by assuming different initial rotation velocities. Standard (i.e., non-rotating) predictions are also shown. The subgiant stars (with $T_{\text {eff }}$ between $\sim$ 6830 and $5800 \mathrm{~K}$ ) are compared with the $1.8 M_{\odot}$ model predictions, while the relevant tracks for the giants (with $T_{\text {eff }}$ at $\sim 4900$ $5000 \mathrm{~K}$ ) are the $2 M_{\odot}$ ones.

In the classical (non-rotating) case, the surface depletion of both $\mathrm{Li}$ and $\mathrm{Be}$ begins relatively late in $T_{\text {eff }}$ within the Hertzsprung gap (i.e., at $\sim 5500 \mathrm{~K}$ ). This depicts dilution of the external layers with nuclear-processed material when the convective stellar envelope deepens in mass. However and as shown in Fig. 13, actual dilution starts earlier than predicted classically, as already revealed by Li data both in open clusters like IC 4651 (Pasquini et al. 2004) and NGC 3680 (Pasquini et al. 2001), but also in field stars (Alschuler 1975; Brown et al. 1989; Lèbre et al. 1999; Do Nascimento et al. 2000; Palacios et al. 2003). As can be seen in Fig. 13, the Be depletion also occurs earlier than predicted in the classical case. This simply means that the Li- and Be-free regions are larger in real main sequence stars than classically predicted.

This favors the complete hydrodynamical models, since rotation-induced mixing acting on the main sequence enlarges the $\mathrm{Li}$ - and $\mathrm{Be}$-free regions inside the stars, implying earlier (i.e., at higher $T_{\text {eff }}$ inside the Hertzsprung gap) Li and Be depletion at the surface of subgiant stars, as well as lower Li and Be values after dredge-up in giant stars (see also Palacios et al. 2003, for model comparison with Li data for field stars). The behavior of both elements predicted in the rotating case nicely matches the data for the subgiant and giant stars in IC 4651, and a dispersion in initial rotation velocity explains the observed abundance dispersion along the evolutionary sequence well.

Lithium abundances of the red giants of IC 4651 are discussed in detail by Pasquini et al. (2004). As can be seen from Table 2, Li was detected in only two giants, although all five of them have very similar atmospheric parameters. To explain

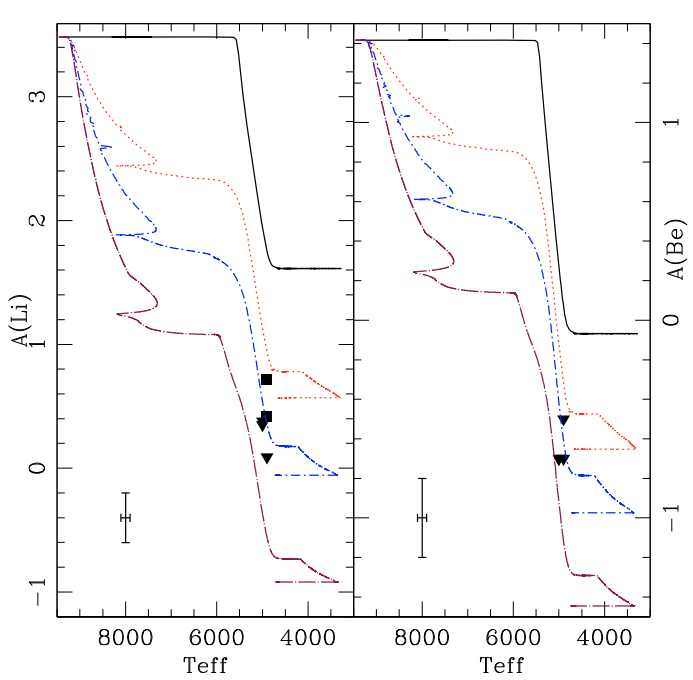

Fig. 14. $\mathrm{Li}$ and Be abundances in IC 4651 evolved stars brighter than $\mathrm{V} \sim 12.6$. Predictions are shown for the $2 M_{\odot}$ model. The solid line is for the classical case, while the other lines present the results for the rotating models computed with different initial rotation velocities (110, 180 , and $250 \mathrm{~km} \mathrm{~s}^{-1}$ for dotted, and dot-dashed, and dot-long dashed lines, respectively). The Li tracks are shifted by 0.16 dex as explained in the text.

this, Pasquini et al. (2004) suggests that the Li observed in these stars might have been produced in their interior after the first dredge-up. We can see, however, that this is not necessary, since in the corresponding stellar mass domain, differences in initial rotation velocities are expected to lead naturally to a significant dispersion $(\sim 1.6$ dex for the range in initial rotation assumed here) in post-dredge-up Li values, accounting nicely for the observed data.

Pasquini et al. (2004) also discusses the issue of the evolutionary status of the giant stars in IC 4651. Their proper classification is based only on their position at the CMD and is not straightforward. These stars could indeed be either on the first ascent RGB or at the clump. As mentioned in Sect. 4.1.1, the new models presented here are computed to include thermohaline mixing following Charbonnel \& Zahn (2007). This process only becomes efficient when the stars reach the bump on the RGB. It then leads to an additional decrease in both the $\mathrm{Li}$ and Be surface abundance at $T_{\text {eff }} \sim 4250 \mathrm{~K}$, as can be seen in Fig. 14. Clump stars are thus expected to have lower $\mathrm{Li}$ and $\mathrm{Be}$ surface abundances than their RGB counterparts. However, for a $2 M_{\odot}$ star the effect is relatively modest (see also Charbonnel \& Lagarde, in preparation), and the theoretical predictions cannot help us disentangling the evolutionary status of the giants.

\section{Conclusions}

Beryllium abundances were calculated for twenty-one main sequence, turn-off, subgiant, and giant stars belonging to the open cluster IC 4651. This is the first time that such an analysis has been carried out along the whole evolutionary sequence of an open cluster.

The Be abundances are found to closely follow the behavior of the Li abundances as determined by Pasquini et al. (2004). The coolest main sequence stars observed present no Be abundance dispersion, and a well-defined Be dip overlaps the welldocumented Li dip for F-type stars. The Be abundances of postmain-sequence stars confirm that first-dredge up dilution starts 
earlier than the expected classically and present a significant dispersion. This is consistent with the Li behavior in evolved stars in IC 4651, in NGC 3680, and in the field.

Our observations are compared with theoretical predictions. For the main sequence and turnoff stars lying on the hot side of the Li-dip, as well as for the evolved stars, we compared the observations with results of new hydrodynamical stellar models calculated with STAREVOL V3.1 (Charbonnel \& Lagarde, in preparation). These models include the effects of atomic diffusion, meridional circulation, shear turbulence, and thermohaline mixing. (Wave-induced transport is negligible in the case of these stars that have very narrow convective envelope while on the main sequence.) For the main sequence stars lying on the cool side of the Li dip, we compared the observations with the $1.2 M_{\odot}$ model of Charbonnel \& Talon (2005) which also includes the transport of angular momentum by internal gravity waves.

The models reproduce all the $\mathrm{Li}$ and Be features very nicely along the CMD of IC 4651. The dispersion of Li and Be abundances on the blue side of the dip and in evolved stars is very well explained when accounting for a dispersion in the initial values of the stellar rotational velocity as observed in young open clusters. On the other hand, the lack of abundance dispersion in lower mass stars is expected to be caused by the impact of internal gravity waves.

The success in explaining the $\mathrm{Li}$ and $\mathrm{Be}$ abundances along the whole evolutionary sequence of IC 4651 is very encouraging. It shows that important steps have been taken towards the proper understanding of the physical mechanisms acting during the stellar evolution.

Acknowledgements. This research has made use of the WEBDA database, operated at the Institute for Astronomy of the University of Vienna, of NASA's Astrophysics Data System, and of the Simbad database operated at CDS, Strasbourg, France. R.S. acknowledges FAPESP PhD (04/13667-4) and post-doc fellowships (08/55923-8). C.C. and N.L. acknowledge financial support from the FNS (Switzerland) and from the Programme National de Physique Stellaire of CNRS/INSU (France).

\section{References}

Allende Prieto, C., Lambert, D. L., \& Asplund, M. 2001, ApJ, 556, L63

Alonso, A., Arribas, S., \& Martinez-Roger, C. 1996, A\&AS, 117

Alonso, A., Arribas, S., \& Martínez-Roger, C. 1999, A\&AS, 140, 261

Alschuler, W. R. 1975, ApJ, 195, 649

Anthony-Twarog, B. J., Mukherjee, K., Twarog, B. A., \& Caldwell, N. 1988, AJ, 95,1453

Asplund, M. 2004, A\&A, 417, 769

Asplund, M., Grevesse, N., \& Sauval, A. J. 2006, Nucl. Phys. A, 777, 1

Balachandran, S. 1995, ApJ, 446, 203

Balachandran, S. C., \& Bell, R. A. 1998, Nature, 392, 791

Biazzo, K., Pasquini, L., Girardi, L., et al. 2007, A\&A, 475, 981

Boesgaard, A. M. 1976, ApJ, 210, 466

Boesgaard, A. M., \& King, J. R. 2002, ApJ, 565, 587

Boesgaard, A. M., \& Krugler Hollek, J. 2009, ApJ, 691, 1412

Boesgaard, A. M., \& Tripicco, M. J. 1986, ApJ, 302, L49

Boesgaard, A. M., Heacox, W. D., \& Conti, P. S. 1977, ApJ, 214, 124

Boesgaard, A. M., Armengaud, E., \& King, J. R. 2004a, ApJ, 605, 864

Boesgaard, A. M., Armengaud, E., King, J. R., Deliyannis, C. P., \& Stephens, A. 2004b, ApJ, 613, 1202

Brown, J. A., Sneden, C., Lambert, D. L., \& Dutchover, E. J. 1989, ApJS, 71, 293

Burkhart, C., \& Coupry, M. F. 1998, A\&A, 338, 1073

Burkhart, C., \& Coupry, M. F. 2000, A\&A, 354, 216

Carretta, E., Bragaglia, A., Gratton, R. G., \& Tosi, M. 2004, A\&A, 422, 951

Castelli, F., \& Kurucz, R. L. 2003, in Proc. IAU Symp. 210, ed. N. Piskunov, W. W. Weiss, \& D. F. Gray, A20

Cayrel, R., Cayrel de Strobel, G., Campbell, B., \& Dappen, W. 1984, ApJ, 283, 205
Charbonnel, C., \& Talon, S. 1999, A\&A, 351, 635

Charbonnel, C., \& Talon, S. 2005, Science, 309, 2189

Charbonnel, C., \& Talon, S. 2008, in Proc. IAU Symp. 252, ed. L. Deng \& K. L. Chan, 163-174

Charbonnel, C., \& Zahn, J.-P. 2007, A\&A, 467, L15

Charbonnel, C., Vauclair, S., \& Zahn, J.-P. 1992, A\&A, 255, 191

Chen, Y. Q., Nissen, P. E., Benoni, T., \& Zhao, G. 2001, A\&A, 371, 943

Chmielewski, Y., Brault, J. W., \& Mueller, E. A. 1975, A\&A, 42, 37

Coelho, P., Barbuy, B., Meléndez, J., Schiavon, R. P., \& Castilho, B. V. 2005, A\&A, 443, 735

Cunha, K., Hubeny, I., \& Lanz, T. 2006, ApJ, 647, L143

Decressin, T., Mathis, S., Palacios, A., et al. 2009, A\&A, 495, 271

Dekker, H., D’Odorico, S., Kaufer, A., Delabre, B., \& Kotzlowski, H. 2000, in Optical and IR Telescope Instrumentation and Detectors, ed. M. Iye \& A. F. Moorwood, Proc. SPIE, 4008, 534

Deliyannis, C. P., \& Pinsonneault, M. H. 1997, ApJ, 488, 836

Deliyannis, C. P., Boesgaard, A. M., Stephens, A., et al. 1998, ApJ, 498, L147

Deliyannis, C. P., Pinsonneault, M. H., \& Charbonnel, C. 2000, in Proc. IAU Symp. 198, ed. L. da Silva, R. De Medeiros, \& M. Spite, 61

Do Nascimento, Jr., J. D., Charbonnel, C., Lèbre, A., de Laverny, P., \& De Medeiros, J. R. 2000, A\&A, 357, 931

Duncan, D. K. 1981, ApJ, 248, 651

Eggen, O. J. 1971, ApJ, 166, 87

Gaigé, Y. 1993, A\&A, 269, 267

Garcia Lopez, R. J., \& Spruit, H. C. 1991, ApJ, 377, 268

Garcia Lopez, R. J., Rebolo, R., \& Perez de Taoro, M. R. 1995, A\&A, 302, 184

García Pérez, A. E., \& Primas, F. 2006, A\&A, 447, 299

Goldreich, P., Murray, N., \& Kumar, P. 1994, ApJ, 424, 466

Grevesse, N., \& Sauval, A. J. 1998, Space Sci. Rev., 85, 161

Herbig, G. H. 1965, ApJ, 141, 588

Hobbs, L. M., Iben, I. J., \& Pilachowski, C. 1989, ApJ, 347, 817

Jones, B. F., Fischer, D., Shetrone, M., \& Soderblom, D. R. 1997, AJ, 114, 352

Jones, B. F., Fischer, D., \& Soderblom, D. R. 1999, AJ, 117, 330

Kawaler, S. D. 1988, ApJ, 333, 236

Lambert, D. L., \& Reddy, B. E. 2004, MNRAS, 349, 757

Lèbre, A., de Laverny, P., De Medeiros, J. R., Charbonnel, C., \& da Silva, L. 1999, A\&A, 345, 936

Lodders, K. 2003, ApJ, 591, 1220

Maeder, A., \& Zahn, J.-P. 1998, A\&A, 334, 1000

Meibom, S. 2000, A\&A, 361, 929

Meibom, S., Andersen, J., \& Nordström, B. 2002, A\&A, 386, 187

Michaud, G. 1986, ApJ, 302, 650

Michaud, G., Richard, O., Richer, J., \& VandenBerg, D. A. 2004, ApJ, 606, 452

Montalbán, J., \& Schatzman, E. 2000, A\&A, 354, 943

Müller, E. A., Peytremann, E., \& de La Reza, R. 1975, Sol. Phys., 41, 53

Pace, G., Pasquini, L., \& François, P. 2008, A\&A, 489, 403

Palacios, A., Talon, S., Charbonnel, C., \& Forestini, M. 2003, A\&A, 399, 603

Paquette, C., Pelletier, C., Fontaine, G., \& Michaud, G. 1986, ApJS, 61, 177

Pasquini, L., Liu, Q., \& Pallavicini, R. 1994, A\&A, 287, 191

Pasquini, L., Randich, S., \& Pallavicini, R. 1997, A\&A, 325, 535

Pasquini, L., Randich, S., \& Pallavicini, R. 2001, A\&A, 374, 1017

Pasquini, L., Randich, S., Zoccali, M., et al. 2004, A\&A, 424, 951

Primas, F., Duncan, D. K., Pinsonneault, M. H., Deliyannis, C. P., \& Thorburn, J. A. 1997, ApJ, 480, 784

Randich, S., Primas, F., Pasquini, L., \& Pallavicini, R. 2002, A\&A, 387, 222

Randich, S., Primas, F., Pasquini, L., Sestito, P., \& Pallavicini, R. 2007, A\&A, 469,163

Santos, N. C., Israelian, G., Randich, S., García López, R. J., \& Rebolo, R. 2004, A\&A, 425, 1013

Sestito, P., \& Randich, S. 2005, A\&A, 442, 615

Smiljanic, R., Pasquini, L., Primas, F., et al. 2008, MNRAS, 385, L93

Smiljanic, R., Gauderon, R., North, P., et al. 2009a, A\&A, 502, 267

Smiljanic, R., Pasquini, L., Bonifacio, P., et al. 2009b, A\&A, 499, 103

Soderblom, D. R., Oey, M. S., Johnson, D. R. H., \& Stone, R. P. S. 1990, AJ, 99, 595

Stephens, A., Boesgaard, A. M., King, J. R., \& Deliyannis, C. P. 1997, ApJ, 491, 339

Swenson, F. J., \& Faulkner, J. 1992, ApJ, 395, 654

Talon, S., \& Charbonnel, C. 1998, A\&A, 335, 959

Talon, S., \& Charbonnel, C. 2003, A\&A, 405, 1025

Talon, S., \& Charbonnel, C. 2005, A\&A, 440, 981

Talon, S., \& Zahn, J.-P. 1997, A\&A, 317, 749

Théado, S., \& Vauclair, S. 2003, ApJ, 587, 795

Wallerstein, G., Herbig, G. H., \& Conti, P. S. 1965, ApJ, 141, 610

Young, P. A., Knierman, K. A., Rigby, J. R., \& Arnett, D. 2003, ApJ, 595, 1114

Zahn, J.-P. 1992, A\&A, 265, 115

Zappala, R. R. 1972, ApJ, 172, 57 